\title{
Numerical Simulation of Anisotropic Shielding of Weak Magnetic Fields
}

\author{
Eugeniusz Kurgan \\ AGH University of Science and Technology, Department of Electrical Engineering, \\ al. Mickiewicza 30, 30-059 Krakow, Poland, \\ kurganaagh.edu.pl
}

\begin{abstract}
In this paper the method of computation of weak magnetic fields in the presence of anisotropic shields it is described. The formulation is based on vector magnetic potential and finite element formulation. Investigation of influence of anisotropic ratio on shielding effectiveness of low level magnetic fields is investigated. At the end some illustrative example in 3D is given and numerical results are compared with experimental data.
\end{abstract}

\section{Introduction}

In the last time there is an increasing interest in low-frequency magnetic shielding. Generally, the shielding effectiveness for low-frequency fields can be obtained by solving Maxwell's equations with appropriate assumptions and boundary conditions [1]. However, the complexity of real shield and source geometries and the anisotropy of the medium do not allow a solution to be easily obtained, unless numerical methods are exploited. Furthermore, even if an analytical solution can be achieved, it might be so complex to be of no practical use for shielding design. Nevertheless, the analysis of magnetic shields by standard numerical methods, for example, the finite element method, gives sufficient tool for design of practical shields, especially when the number of layers is low [2,3].

One means for reducing magnetic fields in given region is to make use of some properties of materials, as a way for altering the spatial distribution of such fields from field source. When a shielding magnetic material separates wires with currents, that are sources for a magnetic field from regions, where reduction of field induction $\mathbf{B}$ is required, the shielding sheets cause a change in the distribution of the magnetic field, directing lines of magnetic flux away from the shielded domain [4].

A quantitative measure of the magnetic shield in reducing the magnetic induction at a given place is the shielding coefficient. It is defined as the ratio of magnitude of magnetic induction at given point when the shield is present, to the magnitude of magnetic induction at the same point, when the shielding material is absent. In general shielding factor is a function of material properties, position at which it is measured, distance of the shield from the field source and magnitude of the excitation [5]. If the magnetic permeability of a shielding material depends significantly from flux 
density within magnetic material, the shielding factor is dependent on excitation value. Problem geometry also plays very important role, both from theoretical and practical point of view [3].

Special difficulties arise in the case of shielding of weak magnetic fields. As it was pointed in [1] by induction of the order of $10 \mu \mathrm{T}$ ferromagnetic materials behave fast as paramagnetics. As a result shielding effectiveness becomes very low. In [1] author suggested to use anisotropy effect to increase shielding coefficient. He gives experimental data which show that such shielding method for very weak fields can be to some extend effective.

In this paper author describes numerical simulation of anisotropic shielding of weak electromagnetic fields in the case of magnetic field. The formulation is based on vector magnetic potential. At the end some illustrative example is given.

\section{Main Equations}

Material equation for anisotropic magnetic material can be written in general form as:

$$
\mathbf{B}=\stackrel{\bar{\mu}}{\mathbf{H}}
$$

When spatial coordinate axes are chosen to be coincident with main anisotropic axes of the magnetic material, the constitutive relation (1) has the form:

$$
\left[\begin{array}{l}
B_{x} \\
B_{y} \\
B_{z}
\end{array}\right]=\left[\begin{array}{ccc}
\mu_{1} & 0 & 0 \\
0 & \mu_{2} & 0 \\
0 & 0 & \mu_{3}
\end{array}\right] \cdot\left[\begin{array}{l}
H_{x} \\
H_{y} \\
H_{z}
\end{array}\right]=\left[\begin{array}{l}
\mu_{1} H_{x} \\
\mu_{2} H_{y} \\
\mu_{3} H_{z}
\end{array}\right]
$$

It is assumed here that anisotropic axes overlap geometrical axes. Here $\mu_{1}, \mu_{2}$ and $\mu_{3}$ are permeability coefficients for $x, y$, and $z$ axes, respectively. This equation can be written in simpler form as:

$$
B_{x}=\mu_{1} H_{x} \quad B_{y}=\mu_{2} H_{y} \quad B_{z}=\mu_{3} H_{z}
$$

After introducing vector magnetic material $\mathbf{A}$ given by

$$
\mathbf{B}=\operatorname{rot} \mathbf{A}
$$

and utilizing equations (3), vector components of magnetic field strength $\mathbf{H}$ have the form:

$$
\begin{aligned}
& H_{x}=\frac{1}{\mu_{1}}\left(\frac{\partial A_{z}}{\partial y}-\frac{\partial A_{y}}{\partial z}\right) \\
& H_{y}=\frac{1}{\mu_{2}}\left(\frac{\partial A_{x}}{\partial z}-\frac{\partial A_{z}}{\partial x}\right)
\end{aligned}
$$




$$
H_{z}=\frac{1}{\mu_{3}}\left(\frac{\partial A_{y}}{\partial x}-\frac{\partial A_{x}}{\partial y}\right)
$$

Ampere's law

$$
\operatorname{rot} \mathbf{H}=\mathbf{J}
$$

gives relation between vector components of magnetic field strength and current density vector

$$
\begin{aligned}
& \frac{\partial H_{z}}{\partial y}-\frac{\partial H_{y}}{\partial z}=J_{x} \\
& \frac{\partial H_{x}}{\partial z}-\frac{\partial H_{z}}{\partial x}=J_{y} \\
& \frac{\partial H_{y}}{\partial x}-\frac{\partial H_{x}}{\partial y}=J_{z}
\end{aligned}
$$

Introducing into above equations usual gauge condition for magnetostatic field [8]:

$$
\frac{\partial A_{x}}{\partial x}+\frac{\partial A_{y}}{\partial y}+\frac{\partial A_{z}}{\partial z}=0
$$

we get the final equations for anisotropic case:

$$
\begin{aligned}
& \frac{\partial}{\partial x}\left[\frac{1}{\mu_{2}}\left(\frac{\partial A_{x}}{\partial x}\right)\right]+ \frac{\partial}{\partial y}\left[\frac{1}{\mu_{3}}\left(\frac{\partial A_{x}}{\partial y}\right)\right]+ \\
&+\frac{\partial}{\partial z}\left[\frac{1}{\mu_{2}}\left(\frac{\partial A_{x}}{\partial z}\right)\right]+\frac{\partial}{\partial x}\left[\left(\frac{1}{\mu_{2}}-\frac{1}{\mu_{3}}\right)\left(\frac{\partial A_{y}}{\partial y}\right)\right]=-J_{x} \\
& \frac{\partial}{\partial x}\left[\frac{1}{\mu_{3}}\left(\frac{\partial A_{y}}{\partial x}\right)\right]+\frac{\partial}{\partial y}\left[\frac{1}{\mu_{3}}\left(\frac{\partial A_{y}}{\partial y}\right)\right]+ \\
&+\frac{\partial}{\partial z}\left[\frac{1}{\mu_{1}}\left(\frac{\partial A_{y}}{\partial z}\right)\right]+\frac{\partial}{\partial y}\left[\left(\frac{1}{\mu_{3}}-\frac{1}{\mu_{1}}\right)\left(\frac{\partial A_{z}}{\partial z}\right)\right]=-J_{y} \\
& \frac{\partial}{\partial x}\left[\frac{1}{\mu_{2}}\left(\frac{\partial A_{z}}{\partial x}\right)\right]+\frac{\partial}{\partial y}\left[\frac{1}{\mu_{1}}\left(\frac{\partial A_{z}}{\partial y}\right)\right]+ \\
&+\frac{\partial}{\partial z}\left[\frac{1}{\mu_{1}}\left(\frac{\partial A_{z}}{\partial z}\right)\right]+\frac{\partial}{\partial z}\left[\left(\frac{1}{\mu_{1}}-\frac{1}{\mu_{2}}\right)\left(\frac{\partial A_{x}}{\partial x}\right)\right]=-J_{z}
\end{aligned}
$$


To solve the last three partial differential equations for vector potential $\mathbf{A}$, standard Galerkin's nodal finite element method was used [5].

\section{An Illustrative Example}

As an illustrative example let us consider a rectangular current carrying wire with rectangular cross section, which is shielded by ferromagnetic plate, as it is given in fig.1. Rectangular wire is $400 \mathrm{~mm}$ wide in both $x$ and $y$ direction and wire cross section has value $10 \times 10 \mathrm{~mm}$. Over the wire in $z$ direction there is a permeable plate, which is $360 \mathrm{~mm}$ away from it.

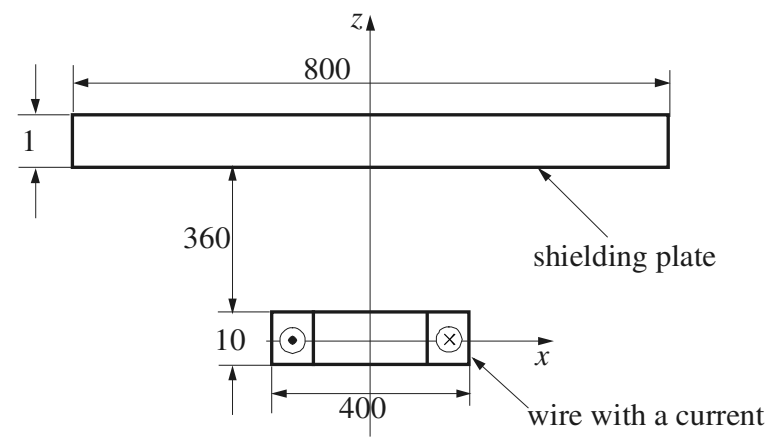

Fig. 1. Geometry description of simple shielding problem. All dimensions are given in $\mathrm{mm}$.

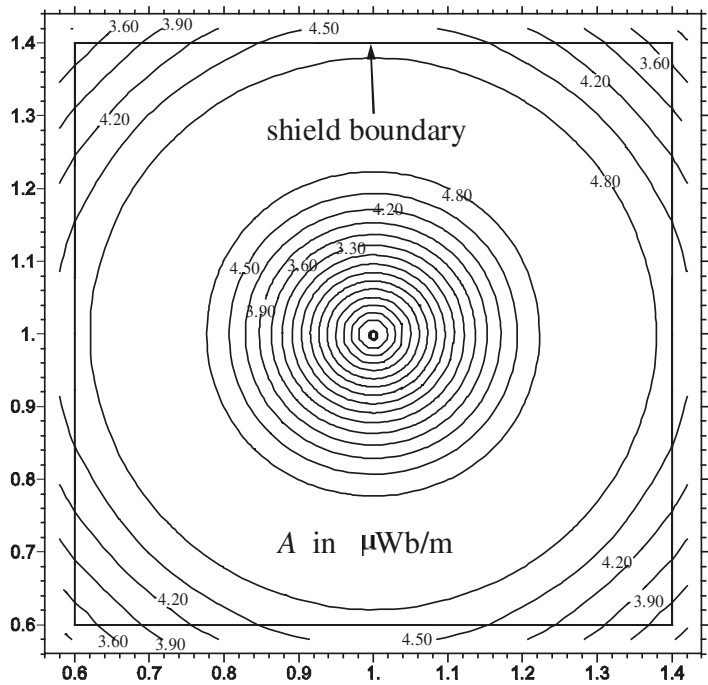

Fig. 2. Equipotential lines of a magnitude of the vector $\mathbf{A} 1 \mathrm{~mm}$ over the plate for $\mu_{1}=5$. 
The pate thickness is $1 \mathrm{~mm}$ and it is $800 \times 800 \mathrm{~mm}$ wide. Total current flowing in the wire has value $200 \mathrm{~A}$, what gives current density $J=200 \mathrm{~A} / \mathrm{cm}^{2}$. Relative permeabilities in $y$ and $z$ direction were in all simulation constant and had values $\mu_{2}=5$ and $\mu_{3}=$ 1 , respectively. In order to explore an influence of anisotropy on magnetic field distribution over the plate only coefficient $\mu_{1}$ was changed. All simulations were carried out for four $\mu_{1}$ values: 5, 35, 70 and 105. In all cases magnetic induction over the shield was less then $60 \mu \mathrm{T}$, what means that only weak magnetostatic fields were considered

Numerical simulation was carried out in full three dimensions where equations (13) to (15) were solved. At distance $2000 \mathrm{~mm}$ from centre of the plate, potential $\mathbf{A}$ and its partial normal derivatives were assumed to be 0 . Whole calculation domain was divided into 14280 tetrahedral finite elements with 67440 functional nodes, what gives altogether 134888 unknowns. These equations were solved by standard iterative method.

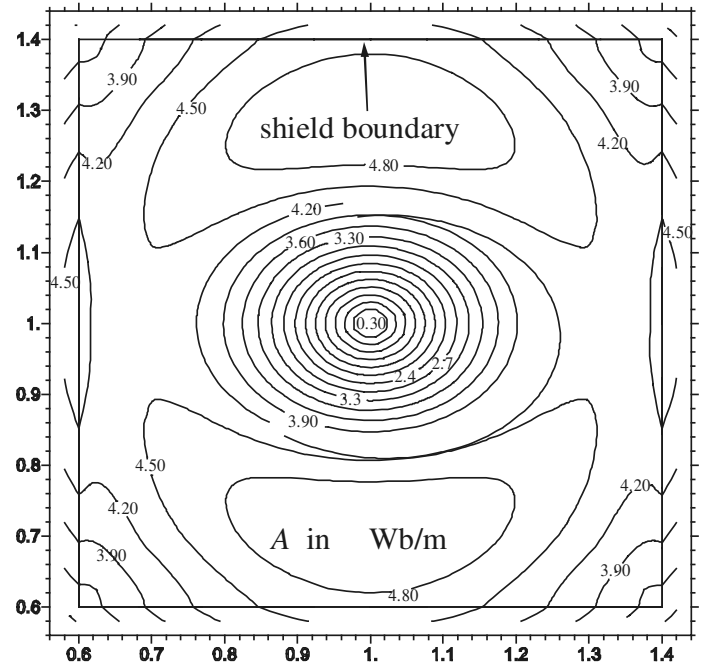

Fig. 3. Equipotential lines of a magnitude of the vector $\mathbf{A} 1 \mathrm{~mm}$ over the plate for $\mu_{1}=70$.

Results of computations are shown in subsequent figures. In figures 2 and 3 equipotential lines of a magnitude of the magnetic vector potential $\mathbf{A}$ are plotted. Fig.2 shows $A$ for relative permeability in $x$ direction $\mu_{1}=5$, that is when material is assumed to be isotropic and in Fig. 3 for $\mu_{1}=70$. In both cases the plots are drawn over a plate at distance $1 \mathrm{~mm}$ in $z$ direction.

In Fig. 2 the equipotantial lines have circular shape cantered at plate middle point while in Fig. 3 one can see deformation of the magnetic field for the anisotropic case. The field is scratched substantially in $x$ direction. 


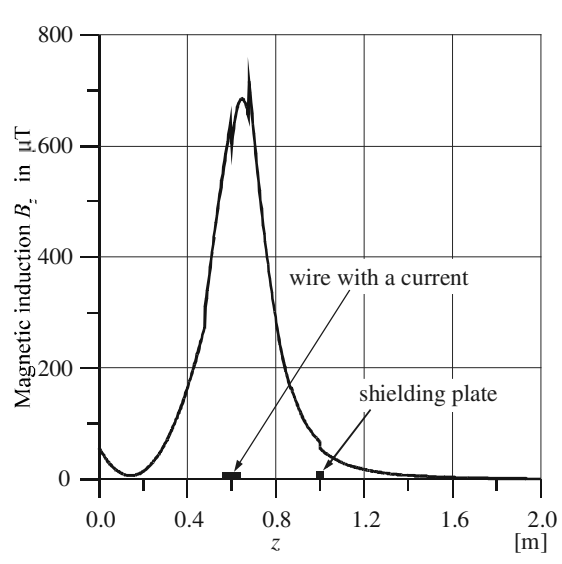

(a)

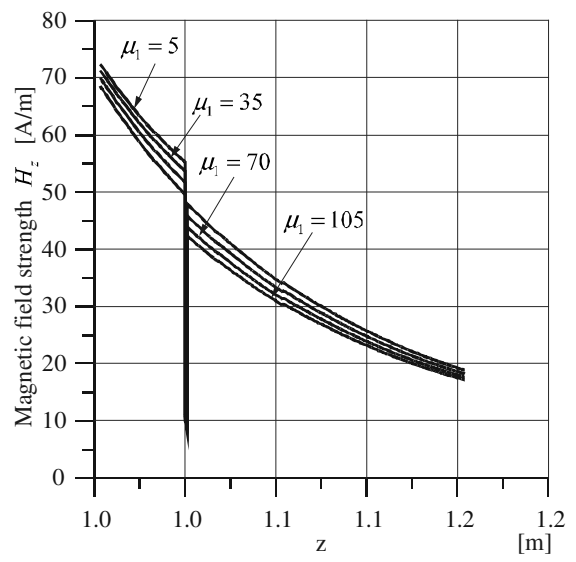

(b)

Fig. 4. Z-component of a magnetic induction $\mathbf{B}$ along $\mathrm{Z}$ axis for relative permeability $\mu_{1}=70$ (a)and z-component of a magnetic field strength $\mathbf{H}$ along $\mathbf{z}$ axis for different values of relative permeability in $x$ direction (b).

Fig.4a shows a plot of $B_{z}$ along $z$ axis for relative permeability $\mu_{1}=70$. On the parallel axis one can see symbolically depicted cross section of current carrying wire and shielding plate. The greatest value of $B_{z}$ is attained in middle of the rectangular wire, as it is to expected. The value of $B_{z}$ near shielding plate is about 100 lower as its maximal value. Over the plate in $z$ direction it decreases substantially. One has to point out that on $z$ axis there is only $z$ component of the field because the $x$ and $y$ components due to problem symmetry are equal zero.

In Fig.4b magnetic field strength $H_{z}$ over the plate in $z$ direction is shown. One can see that for different permeability values the component $H_{z}$ changes not substantially. This is caused by the fact that shield is distant $360 \mathrm{~mm}$ from plate and for this value of the strength, the field permeability is very low. Shielding effectiveness can be increased substantially by placing the shield, where induction is much greater or by increasing plate thickness.

Fig.5a shows plots of $z$ component of a magnetic induction $\mathbf{B}$ over shielding plate along $\mathrm{z}$ axis for different values of relative permeability. One also can see that shielding effectiveness is low and increasing anisotropy does not cause substantial decrease in $B_{z}$.

Fig.5b shows plots of in $B_{z}$ along shielding plate in $x$ direction and the changes of its value for different permabilities. The sharp changes of curves are over the conducting wire. In the magnetostatic case the shielding is due only to flux shunting from the region where decreasing of the field is required. This situation one can observe in figures 2, 3, and 7. Increasing permeability both in $x$ and $y$ directions makes substantially shunting mechanism more effective.

The order of numerical errors can be assessed investigating, how chosen error indicator converges, when number of finite elements and also number of nodes increases. A good candidate for such error indicator can be deduced from Coulomb gauge (17). 


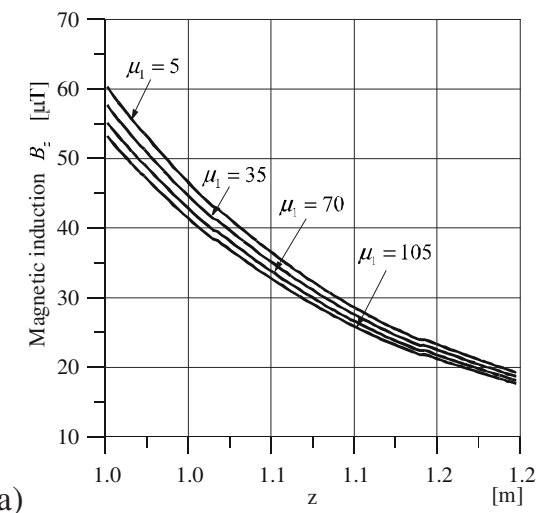

(a)

Fig. 5. Z-component of a magnetic induction $\mathbf{B}$ over shielding plate along $\mathrm{z}$ axis (a) and . $z-$ component of a magnetic induction $\mathbf{B}$ along $\mathrm{z}$ axis (b) for different values of relative permeability.

It states that magnetic vector potential field $\mathbf{A}$ is conservative, what means that in computational domain there are not sources of this field. As consequence in ideal case the total flux of this field through any close surface placed in computational domain should be zero. Thus defining the error indicator in following way:

$$
\text { Error }=\frac{\oint_{S} \mathbf{A} \cdot d \mathbf{s}}{\oint_{S}|\mathbf{A} \cdot \mathbf{n}| \cdot \mathrm{d} s} 100 \%
$$

where $S$ is any closed surface and investigating how it changes with increasing value of number of nodes, we can asses the numerical stability of the computational process. Of course, such defined error indicator is only necessary condition for convergence and not sufficient.

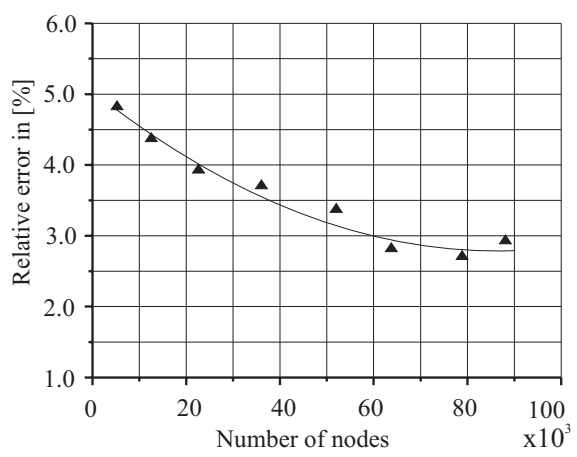

Fig. 6. Relative error indicator given by (16) in function of number of finite element nodes.

Because in shielding plate the filed changes most abruptly, as observation surface $S$ boundary of this plate was chosen. Relative error defined as in (16) is shown in fig. 6 . 
We can see that it decreases as number of elements and nodes increases, what assure us that numerical process is convergent.

\section{Conclusions}

This paper gives descriptive methodology for anisotropic magnetic shielding that is based on solution Maxwell's equations for magnetostatic field in full three dimensions. First equations for vector magnetostatic potential A were formulated and subsequently gauge condition

$$
\operatorname{div} \mathbf{A}=0
$$

implemented. The method is quite general and powerful. It provides a tool for computing the effectiveness of shield design based on anisotropic material properties and geometric dimensions.

General conclusions from all calculation agree with that obtained experimentally in $[6,7]$. The shielding effectiveness for thin shielding plate is rather low.

Acknowledgement. This work was supported by the AGH University of Science and Technology, under grant 11.11.120.183.

\section{References}

1. Magele C.A., Preis K., Renhart W.: Some improvements in non-linear 3D magnetostatics, IEEE Trans. on Magn., vol. 26, (1990) 375-378

2. Ayoub M., Roy F., Bouillault F., Razek A.: Numerical modelling of 3D magnetostatic saturated structures, IEEE Trans. on Magn., vol. 28, (1992)1052-1055

3. Kraehenbuehl, L., Muller D.: Thin layers in electrical engineering. Example of shell models in analysis eddy-currents by boundary and finite element methods, IEEE Trans. on Magn., vol. 29, (1993) 1450-1455

4. Kurgan E.: Magnetic analysis of inhomogeneous double-layer shields at low frequencies, Proc. of the $15^{\text {th }}$ International Symposium on Electromagnetic Compatibility, Wrocław, (2000) $326-330$

5. Silvester P., Ferrari R.L.: Finite elements for electrical engineers, Cambridge University Press, Cambridge, 1996.

6. Karwat, T.: Influence of the anisotropy on the shielding effectiveness of electromagnetic devices. (in Polish), Proc. of XXIV International Seminar on Fundamentals of Electrotechnics and Circuit Theory, Gliwice-Ustroń, (2001) 81 - 84

7. Kurgan E.: Magnetic Shielding of a PCB Stripline Structure, Proc. of Seminar on Electrical Engineering BSE’2001, vol. 13, Istebna, (2001) 106 - 111 